\title{
Utilidad de la tomografía multicorte en el tratamiento quirúrgico del pecho arcuato en niños
}

\author{
Usefulness of multislice tomography for surgical \\ treatment of arcuate chest in children
}

\author{
Carlos García-Hernández,* Lourdes Carvajal-Figueroa,* Christian Archivaldo-García, \\ Carlos Pique-Aragón,* Sergio Landa-Juárez,* Gerardo Izundegui-Ordoñez*
}

*Hospital Infantil Privado; Universidad Nacional Autónoma de México, Ciudad de México.

\begin{abstract}
RESUMEN. Introducción: Para la corrección del pecho arcuato se requiere conocer el ángulo y sitio del corte esternal. El objetivo de este trabajo es mostrar la utilidad de la tomografía de tórax para la planeación de la corrección. Caso clínico: Masculino de 15 años, pecho arcuato desde el nacimiento. En la tomografía se identificaron curvatura, ángulo y distancia al sitio de corrección. Se usaron estas medidas durante la operación. Conclusión: La tomografía preoperatoria permitió medir sitio, ángulo y profundidad del corte esternal, parámetros que se usaron durante la operación, permitiendo una corrección adecuada en el caso de nuestro paciente.
\end{abstract}

Palabras clave: Pecho arcuato, síndrome Currarino Silverman, esternocondroplastía.

\section{INTRODUCCIÓN}

El pecho arcuato, también conocido como pecho de paloma, síndrome condromanubrial o síndrome de CurrarinoSilverman, ${ }^{1,2}$ es una rara deformidad que representa menos del $1 \%$ de los pacientes con pectus carinatum. ${ }^{3}$ Currarino y Silverman describieron que esta malformación se origina por una obliteración prematura del esternón, lo que ocasiona una angulación anormal. ${ }^{4}$ Ésta es una de las características de dicha malformación que ocurre en el ángulo de Lewis, pero también se presenta una deformidad bilateral del segundo

Correspondencia:

\section{Dr. Carlos García-Hernández}

Hospital Infantil Privado, Ciudad de México, México.

Correo electrónico: carloscirped@hotmail.com

Trabajo recibido: 31-V-2020; aceptado: 08-IX-2020.

Citar como: García-Hernández C, Carvajal-Figueroa L, Archivaldo-García C, Pique-Aragón C, Landa-Juárez S, Izundegui-Ordoñez G. Utilidad de la tomografía multicorte en el tratamiento quirúrgico del pecho arcuato en niños. Neumol Cir Torax. 2020;79(3):176-179. https://dx.doi.org/10.35366/96653
ABSTRACT. Introduction: The correction of the arcuate chest requires knowledge of the angle and sternal cut site. The objective of this work is to show the utility of the chest tomography for planning the correction. Case report: Male 15 years old, arcuate chest from birth. The tomography identified curvature, angle and distance to the correction site. These measurements were used during operation. Conclusion: The preoperative tomography allowed to measure the site, angle and depth of the sternal cut, parameters that were used during the operation, allowing for adequate correction in our patient.

Keywords: Arcuate chest, Currarino Silverman syndrome, sternochondroplasty.

al cuarto cartílagos costales. ${ }^{1,4}$ Con frecuencia se establece un diagnóstico erróneo de esta enfermedad y lo consideran como una variante del pectus excavatum, pero en este síndrome no existe depresión esternal y la apariencia visual se debe a la protrusión de los cartílagos costales. ${ }^{1}$ En la actualidad, el tratamiento quirúrgico requiere de los mismos principios establecidos por Ravitch en 1958 y son los siguientes:

a) condrectomías subpericondriales de los cartílagos deformados,

b) esternotomías en cuña transversas,

c) osteosíntesis esternal. ${ }^{5}$

Para lograr una adecuada corrección de la deformidad esternal se requiere que el ángulo y sitio de corte del esternón sea el correcto. ${ }^{1,3,6-8}$ Éste se puede calcular mediante placas laterales de tórax, tomografías simples o incluso mediante la creación de modelos virtuales tridimensionales del esternón. ${ }^{9}$

El objetivo de este trabajo es mostrar la utilidad de la tomografía de tórax para la planeación de la corrección del pecho arcuato en un paciente pediátrico. 


\section{PRESENTACIÓN DEL CASO}

Se trata de un paciente masculino de 15 años, producto de la gesta dos, obtenido por cesárea a las 33 semanas de gestación, por desprendimiento prematuro de placenta tuvo un peso al nacimiento de 2 kilogramos. Presentó malformación anorrectal baja, resuelta con anoplastía en el período neonatal así como hipospadias distal resuelto por uretroplastía a los tres años.

Su padecimiento actual lo inició desde el nacimiento al notar una depresión central en el tórax con protrusión lateral en la parte superior. Este defecto se fue incrementando con el tiempo siendo más notorio en los últimos dos años, en que presentó también dolor y disnea de grandes esfuerzos.

A la exploración física se encontró una severa protrusión del tercio superior del esternón, a nivel del ángulo de Lewis con una aparente depresión del tercio inferior (Figura 1) acompañada de elevación de los cartílagos costales en el arco costal 2 al 4; el resto de la exploración física fue normal.

La tomografía simple de tórax demostró una curvatura de concavidad posterior en la parte superior del esternón (Figura 2), así como la presencia de escoliosis. La valoración cardiológica y el ecocardiograma fueron normales, al igual que las pruebas de función respiratoria.

En la planeación del tratamiento quirúrgico para conocer el sitio, longitud y ángulo de corrección para la osteotomía esternal, se llevaron a cabo las siguientes mediciones en la tomografía computarizada: sitio de mayor curvatura, ángulo para la corrección, profundidad de la osteotomía esternal, distancia del borde superior e inferior del esternón al sitio de corrección (Figura 3).

A través de una incisión media que abarca todo el esternón, los colgajos musculares del pectoral mayor fueron

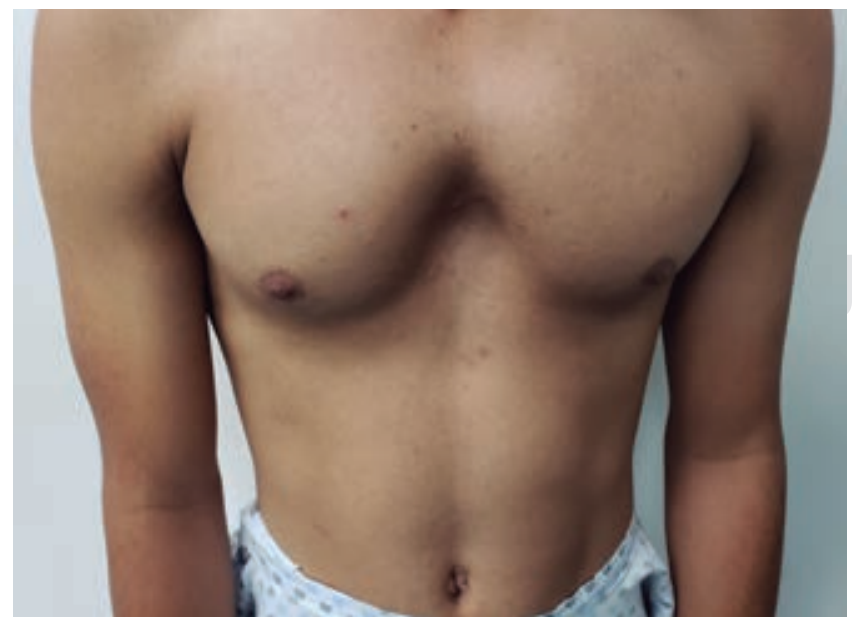

Figura 1: Aspecto clínico característico del pecho arcuato, con protrusión del esternón y de los primeros cartílagos costales.

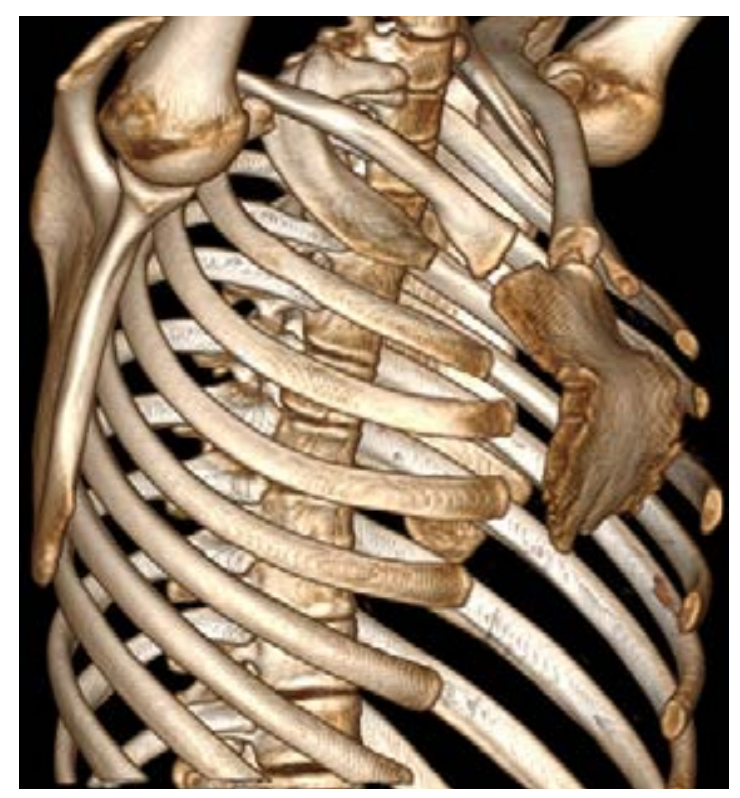

Figura 2: Tomografía simple de tórax que muestra la curvatura anormal en la porción superior del esternón.

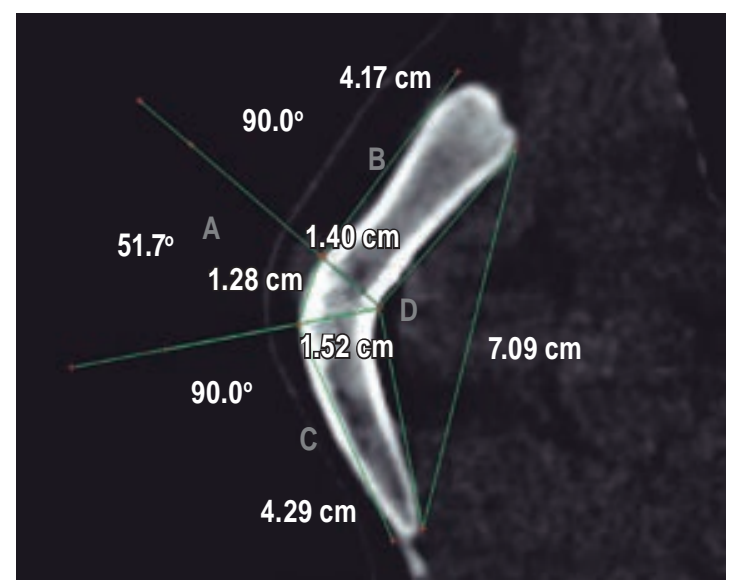

Figura 3: Medición del ángulo requerido de corte para la construcción de la cuña esternal (A), medición del borde superior e inferior (B, C). Profundidad de corte en el esternón (D).

movilizados de manera lateral y separados del esternón y de los cartílagos costales, se practicó a continuación resección subpericondrial del segundo al cuarto cartílago costal. A continuación, se determinó el sitio de las osteotomías de acuerdo con la medición tomográfica, se realizaron dos osteotomías y se resecó la cuña esternal hasta corregir el esternón, se colocaron dos suturas de acero para afrontar el esternón y se estabilizó con una grapa esternal de titanio (Figura 4) con cuatro tornillos sistema Tritium (MR), se afrontaron los pericondrios de los cartílagos costales, se dispuso un drenaje y se procedió al cierre de los planos 
musculares y de la piel. Tiempo quirúrgico de 150 minutos, sin complicaciones ni hemorragia, se consiguió una corrección completa en el posoperatorio inmediato (Figura 5). Se inició vía oral ese mismo día, se retiró drenaje al tercer día del posoperatorio y se egresó sin complicaciones al quinto día. La tomografía de control al décimo día de la operación demostró la corrección completa del esternón (Figura 6).

\section{DISCUSIÓN}

El pecho arcuato, también conocido como síndrome de Currarino-Silverman o pectus carinatum tipo II, es una

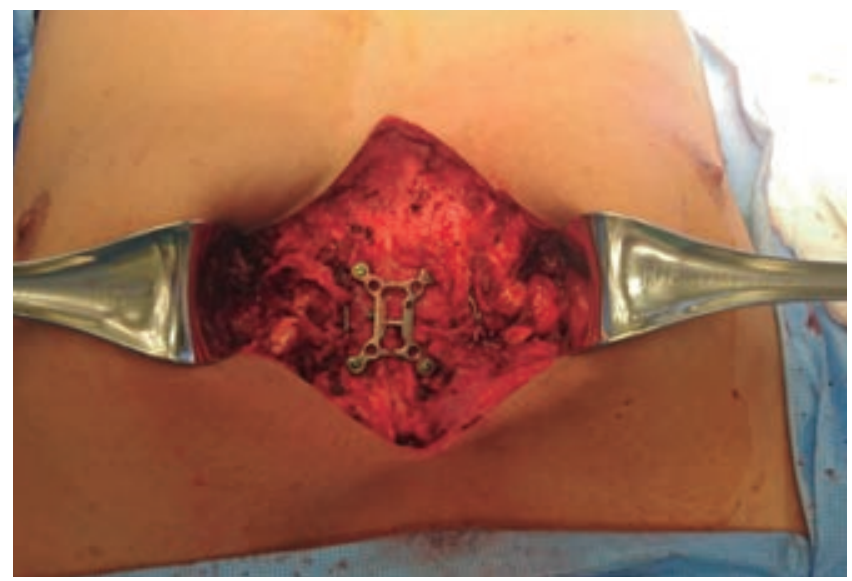

Figura 4: Esternón estabilizado al final de la cirugía con una grapa esternal fijada con cuatro tornillos.

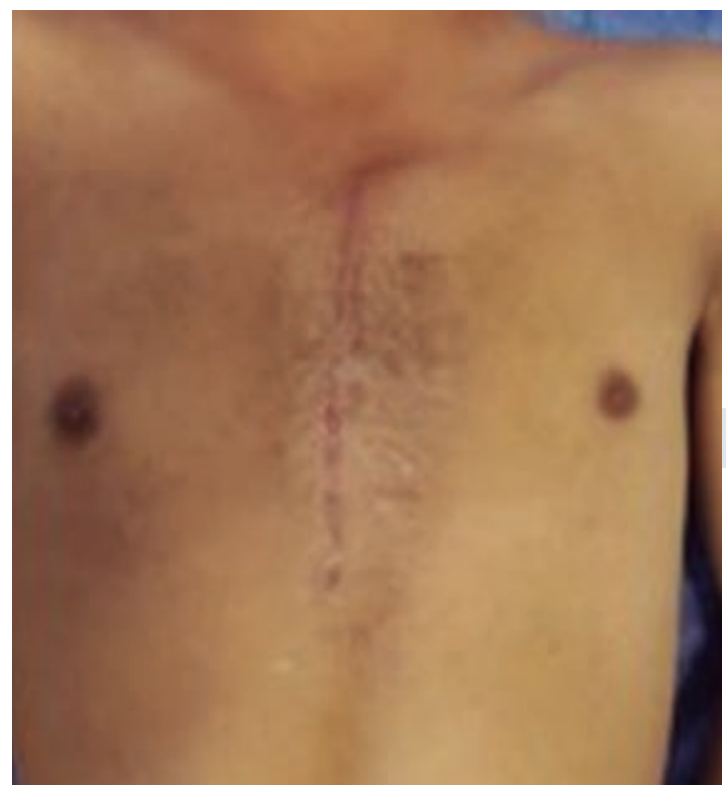

Figura 5: Aspecto posoperatorio un mes después, se aprecia corrección completa de la malformación.

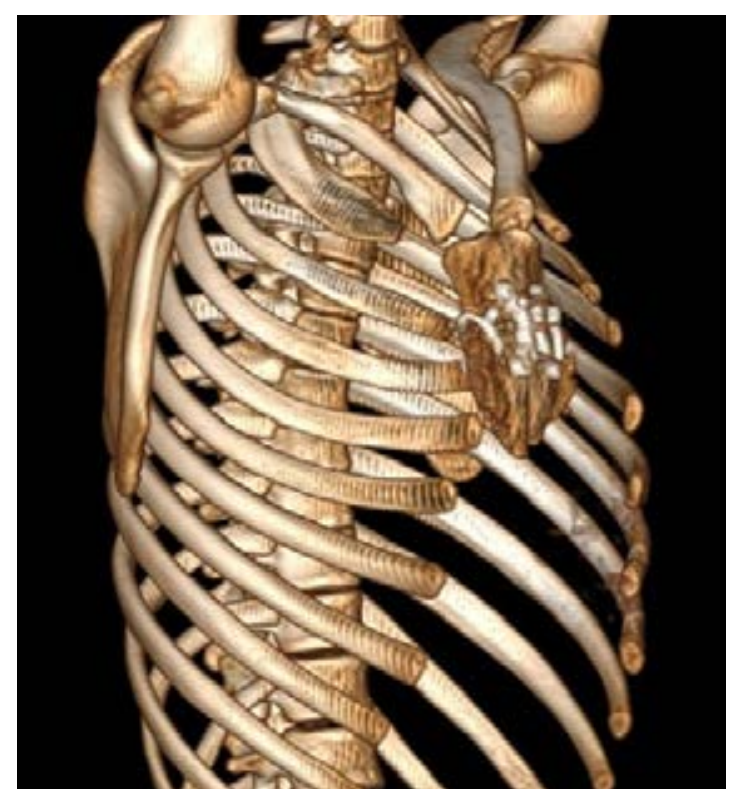

Figura 6: Tomografía posoperatoria. Vista lateral, se aprecia corrección completa de la deformidad esternal.

deformidad poco frecuente de la pared del tórax que con frecuencia es confundida con el pecho excavado., Ésta se caracteriza por una deformidad del esternón a nivel del ángulo de Lewis, así como con una hipertrofia de los cartílagos costales. Esta curvatura anormal desvía el esternón hacia atrás en su parte inferior, además de la hipertrofia de los cartílagos por arriba del ángulo de Lewis que originan la confusión con el pectus excavatum. ${ }^{4}$ Con el crecimiento de cada persona, la deformidad se acentúa y se afecta la morfología y estética de la caja torácica, por lo que está indicada su resolución quirúrgica. Este tratamiento fue descrito por Ravitch en $1958^{5}$ y consiste en la resección de los cartílagos costales deformados preservando el pericondrio, así como osteotomías en el esternón que quiten la curvatura, la cual es diferente en cada paciente. Al final se debe estabilizar el esternón con algún tipo de material de osteosíntesis. Una osteotomía esternal inadecuada puede ocasionar daño mediastinal, así como una corrección inadecuada del esternón. ${ }^{3}$ La tomografía preoperatoria permite conocer la anatomía esternal, así como medir el sitio, ángulo y profundidad del corte esternal, lo que ha permitido el diseño de un molde diseñado de acuerdo con la deformidad esternal, como lo reporta Leng. ${ }^{9}$ En esta investigación medimos la longitud y los ángulos de deformidad del esternón en la tomografía y aplicamos estas medidas durante la operación para determinar sitio, ángulo y profundidad de corte. Esto permitió llevar a cabo una corrección adecuada, lo que pudimos corroborar con la tomografía de control, realizada 15 días después de la operación, en donde apreciamos una corrección adecuada del esternón. 


\section{CONCLUSIONES}

La tomografía computada de tórax es un estudio accesible y de bajo costo que nos permite conocer la anatomía de esta malformación de manera preoperatoria para poder planear una corrección adecuada. Además, es posible realizar este estudio en la mayor parte de los hospitales.

\section{REFERENCIAS}

1. Kuzmichev V, Ershova K, Adamyan R. Surgical correction of pectus arcuatum. J Vis Surg. 2016;2:55. Available from: https://doi. org/10.21037/jovs.2016.02.28

2. Brodkin HA. Congenital chondrosternal prominence (pigeon breast) a new interpretation. Pediatrics. 1949;3(3):286-295.

3. Shamberger RC, Welch KJ. Surgical correction of chondromanubrial deformity (Currarino Silverman syndrome). J Pediatr Surg. 1988;23(4):319322. Available from: https://doi.org/10.1016/s0022-3468(88)80197-0

4. Currarino G, Silverman FN. Premature obliteration of the sternal sutures and pigeon- breast deformity. Radiology. 1958;70(4):532-540. Available from: https://doi.org/10.1148/70.4.532
5. Ravitch MM. Operative correction of pectus carinatum (pigeon breast). Ann Surg. 1960;151(5):705-714. Available from: https://doi. org/10.1097/00000658-196005000-00011

6. Lam CR, Taber RE. Surgical treatment of pectus carinatum. Arch Surg. 1971;103(2):191-196. Available from: https://doi.org/10.1001/ archsurg.1971.01350080107016

7. Brichon PY, Wihlm JM. Correction of a severe pouter pigeon breast by triple sternal osteotomy with a novel titanium rib bridge fixation. Ann Thorac Surg. 2010;90(6):e97-e99. Available from: https://doi. org/10.1016/j.athoracsur.2010.08.068

8. Kim SY, Park S, Kim ER, Park IK, Kim YT, Kang CH. A case of successful surgical repair for pectus arcuatum using chondrosternoplasty. Korean J Thorac Cardiovasc Surg. 2016;49(3):214-217. Available from: https:// doi.org/10.5090/kjtcs.2016.49.3.214

9. Leng S, Bici K, Facchini F, Volpe $Y$, Uccheddu F, Furferi R, et al. Customized cutting template to assist sternotomy in pectus arcuatum. Ann Thorac Surg. 2019;107(4):1253-1258. Available from: https://doi. org/10.1016/j.athoracsur.2018.10.057

Conflicto de intereses: Los autores declaran no tener conflicto de intereses. 